\title{
The Average Surface Temperature in Antarctica
}

\author{
Ruiyang $\mathrm{Li}^{1}$ \\ ${ }^{1}$ College of Renewable Energy, North China Electric Power University, Beijing 102206, China
}

Keywords: The Average Earth Land Surface Temperature, Inverse Distance To A Power Method, Gray Prediction, Hermit Cubic Interpolation, Meshing

\begin{abstract}
The average Earth land surface temperature is a key indicator of climate change and global warming. To estimate the average Earth land surface temperature, we use inverse distance to a power method for spatial interpolation of Antarctica. By observing various automatic weather station data, we estimate that the average surface temperature of the unknown, and then we use gray prediction model for Antarctica mean surface temperature prediction and description. We found that the model error control in $0.2 \%$, with a more accurate fitting resistance, which can be well predicted mean surface temperature in Antarctica.
\end{abstract}

\section{Introduction}

The average Earth land surface temperature is a key indicator of climate change and global warming. Based on the inverse distance to a power method as well as gray prediction model, a mathematical model is able to estimate and define the average surface temperature. Under the premise of considering only Antarctica, we will define and estimate the mean surface temperature.

\section{The Model}

Inverse distance to a power method. First, in order to obtain the boundary of Antarctica, we access to the latitude and longitude coordinates of each boundary point of Antarctica through Google Maps. We transform latitude into the axial length $\left(\boldsymbol{\rho}_{\mathbf{i}}\right)$ and longitude into the polar angle $\left(\boldsymbol{\theta}_{\mathbf{i}}(\mathbf{i}=\mathbf{1}, \mathbf{2}, \ldots, \mathbf{n})\right.$.). We gain the border graphic Antarctica by the establishment of polar coordinates. The range of polar angle is that $(-\boldsymbol{\pi} \sim \boldsymbol{\pi})$, we will divide it( $\boldsymbol{\theta})$ into 10000 parts ,and then we can gain that $\Delta \boldsymbol{\theta}=\frac{\mathbf{2 \pi}}{\mathbf{1 0 0 0 0}}$. We use Hermit cubic interpolation method to get a new set of $\boldsymbol{\rho}_{\mathbf{i}}$ ' corresponded to $\boldsymbol{\theta}_{\mathbf{i}}{ }^{\prime}$. We draw the border of Antarctica through this new point. 


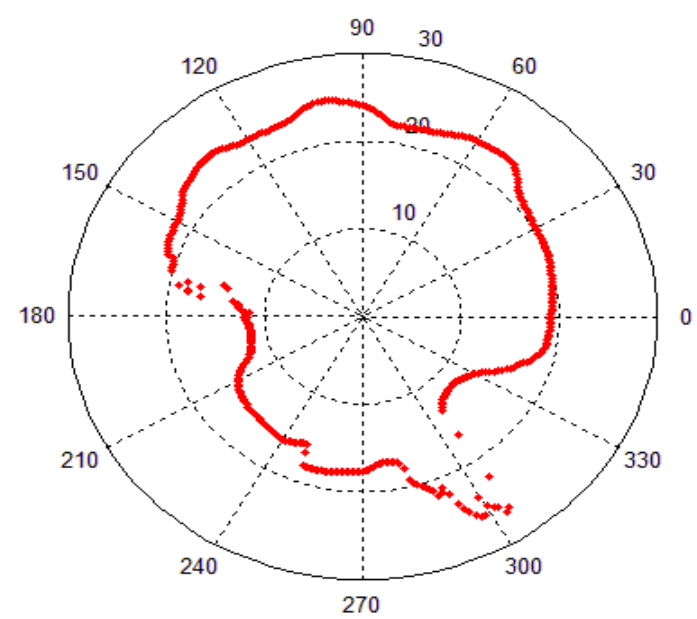

Figure1: Interpolated boundary Antarctica

Simultaneously at the direction of $\rho$, graduation is divided by that $\Delta \rho=0.01$. Thus, the entire graph turns into series of mesh points. According to data from the investigation, we can get a total of $n$ coordinates of the location of meteorological stations $\left(\left(a_{j}, b_{j}\right),(j=1,2, \ldots, n)\right)$. Surface temperature from a known individual weather observations, we estimate the i-th point of the surface temperature .Finally all temperature values that we obtained by calculation are averaged and we can get the entire Antarctic average surface temperature.

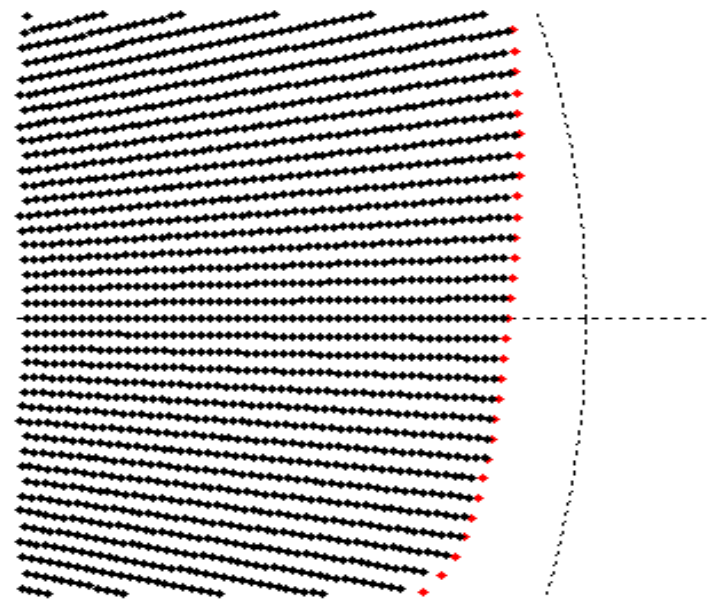

Figure2: Antarctica meshing point results

Using MATLAB, we get solution to the average temperature distribution. It is distribution of Antarctica in the 12 months in 1992-2015. As shown below: 


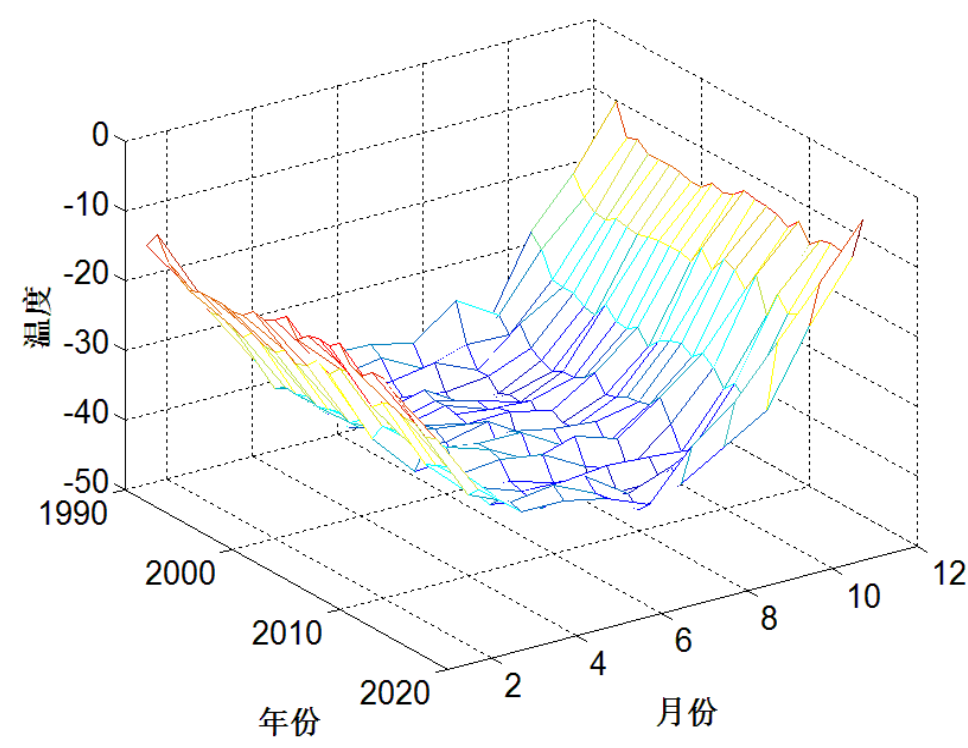

Figure3 :1992--2015 Different month temperature

From the figure, we can find that most of the year around June shows the lowest temperature characteristics. The phenomenon occurs in the Antarctic polar night of the Antarctic between March to September and June is in the middle period of polar night, thus the temperature is colder period.

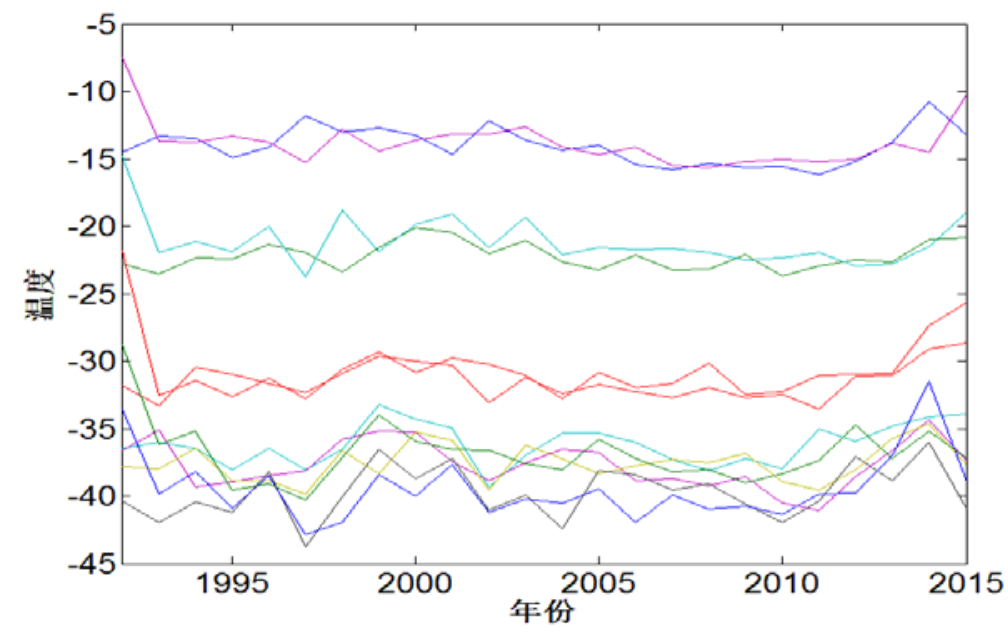

Figure4: January-December the temperature change in different years

We were fitting a straight line to the temperature of each month and gained the following results: we generally find that in March-- September, most of the average temperature shows a downward trend, while in January-March and September-December, average temperature substantially rises. Therefore, we believe that the phenomenon occurs in the Antarctic polar night period, the greenhouse effect do not show. In the very day period, the rise of temperature reflects the result of the greenhouse effect. When polar night happens, we guess that there is no direct sunlight in Antarctica and the greenhouse effect is no way to emerge.

The Gray Prediction Model. We use MATLAB to solve this problem. The data of the year 1992-2015 can be obtained by gray equation, with the original data to make error analysis. The result can be shown in the error figure below. 


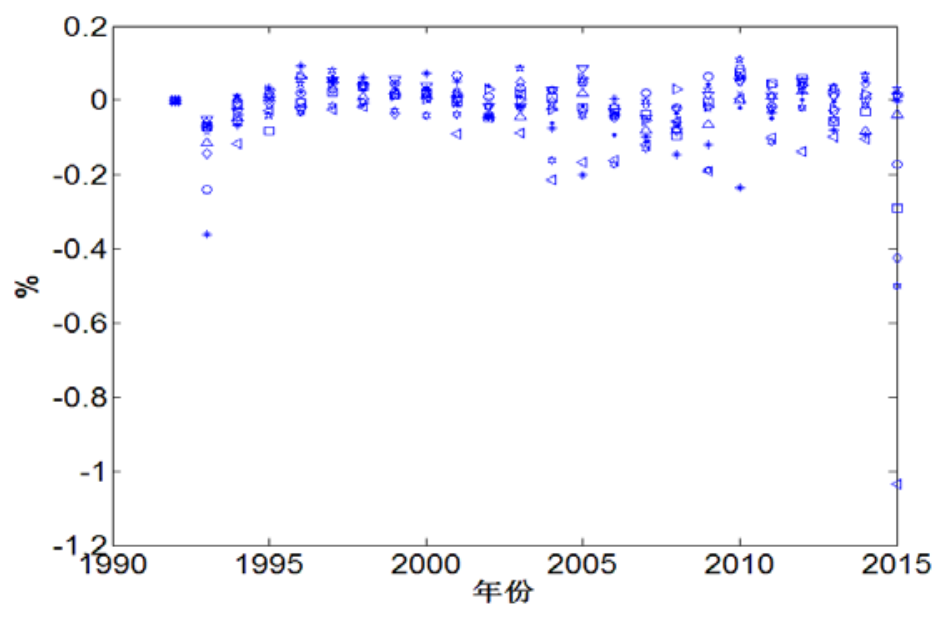

Figure5: Error Figure in different months

From the figure above, we can see that only a few large errors occur in January 2015 data, but most of the errors in the data are distributed in $0.2 \%$, so the use of gray prediction model can get more fitting accurate data.

We also get the data in the next seven years, it can be found that temperature in different months has a similar rule with the mode l. We select the temperature in different months in 2022 to draw temperature curves, as shown below.

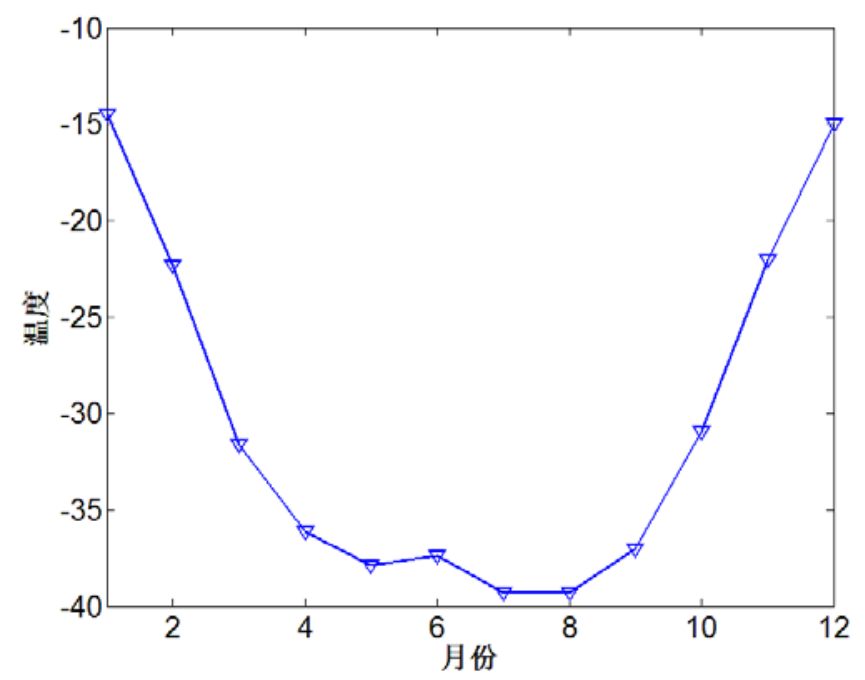

Figure6: Temperature curves in different months in 2022

\section{Conclusion}

By using inverse distance to a power method and gray prediction model, as well as observing various automatic weather station data, we can estimate that the average surface temperature of the unknown, and then we can get the prediction and description of Antarctica mean surface temperature. The model error controls in $0.2 \%$, with a more accurate fitting resistance, which can be well predicted mean surface temperature in Antarctica.

\section{References}

[1] CAI Dihua , GUO Ni , LI Chongwei . Interpolation Method Based on DEM Temperature [J]. 
Arid Meteorology , 2009, 27(1).

[2] HU Fan , CHEN Yizheng . Improved automatic weather stations surface temperature measurement method [J]. Meteorology , 2012,38(3):381-384.

[3] David S M, Moson H H.Using Iterated Function Systems to Model Discrete Sequences [J].IEEE Trans on Processing, 1992, 40(7):1724- 1734.

[4] Zhao C Y, Nan Z G, Cheng G D. Ecological Modeling, 2005, 189: 209.

[5] Zhou S Q, Xue G Y, Gong P. Journal of Geographical Sciences, 2005, 15(2): 223.

[6] Bennett R J. Ann. Assoc. Am. Geogr. Ocean, 1984, 27(3): 521. 